\title{
A quiescent galaxy at the position of the long GRB 050219A $\star, \star \star$
}

\author{
A. Rossi ${ }^{1,2}$, S. Piranomonte ${ }^{3}$, S. Savaglio ${ }^{4,5,6}$, E. Palazzi ${ }^{1}$, M. J. Michałowski ${ }^{7}$, S. Klose ${ }^{2}$, L. K. Hunt ${ }^{8}$, L. Amati ${ }^{1}$, \\ J. Elliott ${ }^{4}$, J. Greiner ${ }^{4}$, C. Guidorzi ${ }^{9}$, J. Japelj ${ }^{10}$, D. A. Kann ${ }^{2}$, B. Lo Faro ${ }^{11}$, A. Nicuesa Guelbenzu ${ }^{2}$, S. Schulze ${ }^{12,13}$, \\ S. D. Vergani ${ }^{14,15}$, L. A. Arnold ${ }^{16}$, S. Covino ${ }^{15}$, V. D’Elia ${ }^{17,3}$, P. Ferrero ${ }^{18}$, R. Filgas ${ }^{19}$, P. Goldoni ${ }^{20}$, \\ A. Küpcü Yoldaş ${ }^{21}$, D. Le Borgne ${ }^{22}$, E. Pian ${ }^{1,23}$, P. Schady ${ }^{4}$, and G. Stratta ${ }^{24}$
}

1 INAF-IASF Bologna, Area della Ricerca CNR, via Gobetti 101, 40129 Bologna, Italy e-mail: a.rossi@iasfbo.inaf.it

2 Thüringer Landessternwarte Tautenburg, Sternwarte 5, 07778 Tautenburg, Germany

3 INAF-Osservatorio Astronomico di Roma, via di Frascati 33, 00040 Monte Porzio Catone, Italy

${ }^{4}$ Max-Planck-Institut für Extraterrestrische Physik, Giessenbachstraße 1, 85748 Garching, Germany

5 Dipartimento di Fisica, Universitá della Calabria, 87036 Arcavacata di Rende, Italy

${ }^{6}$ European Southern Observatory, 85748 Garching, Germany

7 SUPA, Institute for Astronomy, University of Edinburgh, Royal Observatory, Edinburgh, EH9 3HJ, UK

8 INAF-Osservatorio Astrofisico di Arcetri, Largo Fermi 5, 50125 Firenze, Italy

9 Department of Physics and Earth Sciences, University of Ferrara, via Saragat 1, 44122 Ferrara, Italy

10 Faculty of Mathematics and Physics, University of Ljubljana, Jadranska ulica 19, 1000 Ljubljana, Slovenia

11 Aix-Marseille Université, CNRS, LAM (Laboratoire d'Astrophysique de Marseille) UMR 7326, 13388 Marseille, France

12 Instituto de Astrofísica, Pontificia Universidad Católica, Av. Vicuña Mackenna, 4860 Santiago, Chile

13 Millennium Institute of Astrophysics (MAS), Santiago, Chile

14 GEPI, Observatoire de Paris, CNRS, Univ. Paris Diderot, 5 place Jules Jannsen, 92195 Meudon, France

15 INAF - Osservatorio Astronomico di Brera, via E. Bianchi 46, 23807 Merate (LC), Italy

${ }^{16}$ University of Rochester, Department of Physics and Astronomy, Rochester, NY 14627, USA

17 ASI, Science Data Centre, via del Politecnico snc, 00133 Roma, Italy

18 Instituto de Astrofísica de Andalucía (IAA-CSIC), Glorieta de la Astronomía s/n, 1008, Granada, Spain

19 Institute of Experimental and Applied Physics, Czech Technical University in Prague, Horska 3a/22,12800 Prague, Czech Republic

20 APC, Univ. Paris Diderot, CNRS/IN2P3, CEA/Irfu, Obs. de Paris, Sorbonne Paris Cité, 75205 Paris Cedex 13, France

21 Institute of Astronomy, University of Cambridge, Madingley Road CB3 0HA, Cambridge, UK

22 Institut d'Astrophysique de Paris, UMR 7095, CNRS, UPMC Univ. Paris 06, 98bis boulevard Arago, 75014 Paris, France

23 Scuola Normale Superiore di Pisa - Piazza dei Cavalieri 7, 56126 Pisa, Italy

24 Università degli Studi di Urbino "Carlo Bo", Piazza della Repubblica 13, 61029 Urbino, Italy

Received 21 March 2014 / Accepted 28 August 2014

\section{ABSTRACT}

Context. Long-duration gamma-ray bursts (LGRBs) are produced by the collapse of very massive stars. Because of the short life time of their progenitors, LGRBs pinpoint star-forming galaxies. Recent studies demonstrate that LGRBs populate all types of star-forming galaxies from sub-luminous, blue compact dwarfs to luminous infrared galaxies.

Aims. We present here a multi-band search for the host galaxy of the long dark GRB 050219A within the enhanced Swift/XRT error circle. We aim to characterise the properties of its host galaxy and compare them with those of other LGRB host galaxies.

Methods. We used spectroscopic observations acquired with VLT/X-Shooter to determine the redshift and star-formation rate of the most probable host galaxy identified on the basis of a chance probability criterion. We compared the results with the optical and infrared spectral energy distribution obtained with Swift/UVOT, the seven-channel imager GROND at the 2.2-m telescope on La Silla and the Herschel Space Observatory, supplemented by archival observations obtained with FORS2 at the ESO/VLT, the Spitzer Space Telescope, and the GALEX survey.

Results. The most probable host galaxy of the genuine long-duration GRB 050219A is a 3 Gyr-old early-type galaxy at $z=0.211$. It is characterised by a ratio of star-formation rate to stellar mass (specific star-formation rate) of $\sim 6 \times 10^{-12} \mathrm{yr}^{-1}$ that is unprecedentedly low when compared to all known LGRB host galaxies. Its properties resemble those of post-starburst galaxies.

Conclusions. GRB 050219A might be the first known long burst to explode in a quiescent early-type galaxy. This would be further evidence that GRBs can explode in all kinds of galaxies, with the only requirement being an episode of high-mass star formation.

Key words. gamma-ray burst: individual: GRB 050219A

\footnotetext{
* Based on observations collected with GROND at the $2.2 \mathrm{~m}$ telescope of the La Silla Observatory, Chile (PI: Greiner), at the Very Large Telescope of the European Southern Observatory, Paranal, Chile
}

(089.A-0843, PI: Piranomonte), and with the ESA space observatory Herschel (PI: Hunt)

$\star \star$ Appendices are available in electronic form at

http://www. aanda. org 


\section{Introduction}

The gamma-ray burst (GRB) population is divided into two populations based on their duration: long GRBs (LGRBs) and short GRBs (Kouveliotou et al. 1993). LGRBs are associated with the deaths of massive stars mainly because of their association with broad-lined Type-Ic supernovae (e.g., Hjorth et al. 2003; Woosley \& Bloom 2006; Hjorth \& Bloom 2012). Indeed, they can be found in all types of galaxies that feature star-forming activity (GRB host galaxies, GRBHs), from blue and low mass (e.g., Savaglio et al. 2009) to red and massive galaxies (e.g., Rossi et al. 2012; Perley et al. 2013; Hunt et al. 2014).

It has been a matter of debate whether GRBHs might be biased against particular properties such as dust content and metal abundance (e.g., Elliott et al. 2012; Michałowski et al. 2012b; Savaglio et al. 2012; Perley et al. 2013; Graham \& Fruchter 2013; Kelly et al. 2014). Jakobsson et al. (2012) present the redshift distribution of the first optically unbiased LGRB host survey, which included dark LGRBs ${ }^{1}$ (Hjorth et al. 2012). They show that at $z \gtrsim 3$, the LGRB rate does not conform to the conventional determinations of the star-formation rate (SFR) of the universe. To investigate this, Hunt et al. (2014) combined the host samples compiled by Savaglio et al. (2009) and Perley et al. (2013) and improved the sub-sample of the hosts of dark LGRBs by including new data and objects. They find that LGRBs can explode in galaxies with a specific SFR $\left(\mathrm{sSFR}=\mathrm{SFR} / M_{*}\right)$ between $\sim 10^{-10}$ and $\sim 10^{-7} \mathrm{yr}^{-1}$. These values are similar to the more general star-forming galaxy population up to $z \sim 3$ (e.g., Karim et al. 2011).

Here we present a comprehensive study of the putative host galaxy of the long GRB 050219A. In particular we measure its mass and SFR and show that this galaxy has the lowest sSFR in comparison to the GRBHs known so far. Throughout this work, we use a $\Lambda$ CDM world model with $\Omega_{\mathrm{M}}=0.27, \Omega_{\Lambda}=0.73$, and $\mathrm{H}_{0}=71 \mathrm{~km} \mathrm{~s}^{-1} \mathrm{Mpc}^{-1}$ (Spergel et al. 2003). We use magnitudes in the AB system and the Chabrier (2003) initial mass function (IMF). In Appendix A, we analyse the properties of the explosive event and afterglow and show that GRB 050219A is truly a long burst. We also review the evidence that GRB 050219A is a dark burst, and conclude that it satisfies several criteria for such a classification.

\section{Observations}

GRB 050219A triggered the Swift Burst Alert Telescope (BAT, Gehrels et al. 2004; Barthelmy et al. 2005a) at 12:40:01 UT on 19 February 2005 (Hullinger et al. 2005). It was a long burst with a duration of $T_{90}(15-350 \mathrm{keV}) \sim 24 \mathrm{~s}$. The Swift X-ray Telescope (XRT, Burrows et al. 2005) began observing $92 \mathrm{~s}$ after the trigger and found a bright, fading X-ray source (Romano et al. 2005). Simultaneous Swift UV/Optical Telescope (UVOT, Roming et al. 2005) observations started $80 \mathrm{~s}$ after the trigger and did not reveal any new source within the prompt XRT error circle (see Fig. 1). The first ground-based observations were performed $2.05 \mathrm{~h}$ after the event with the Microlensing Observations in Astrophysics (MOA), an $0.6 \mathrm{~m}$ telescope at Mt. John University Observatory, New Zealand. The images did not reveal any variable source down to $R=20.5$ (de Ugarte Postigo et al. 2005; see also Berger \& Gonzalez 2005).

\footnotetext{
1 Here and in the following we consider dark GRBs to be those with an optical afterglow that is observed to be fainter than what is predicted by the extrapolation from the X-rays (e.g., Jakobsson et al. 2004; van der Horst et al. 2009) and following the standard afterglow theory (Sari et al. 1998).
}

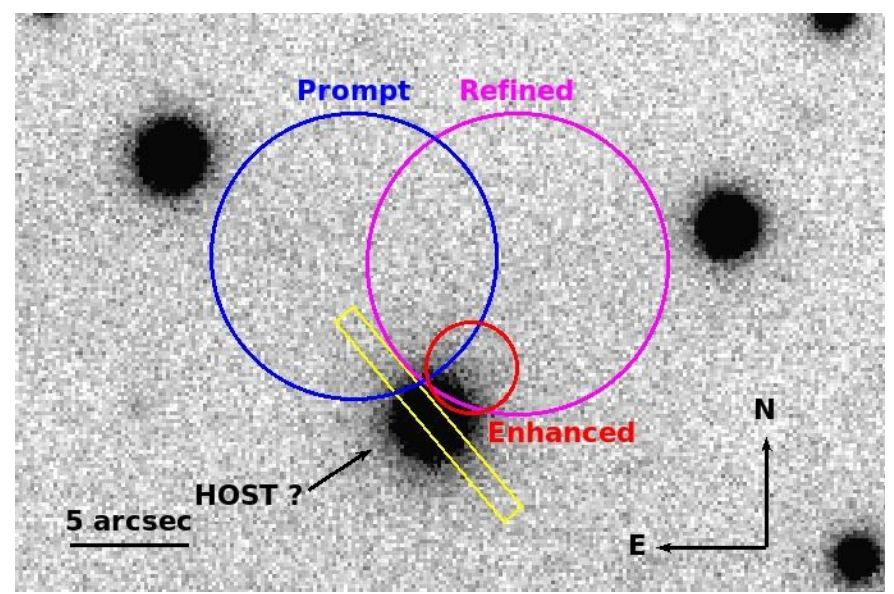

Fig. 1. Zoom into the GROND $r^{\prime}$-band image of the field of GRB 050219A. We highlighted the prompt XRT position (blue), the refined position (magenta) and the final enhanced position (red). The yellow rectangle shows the position of the X-Shooter slit.

Two years later a new enhanced XRT position (Goad et al. 2007; Evans et al. 2009) was published with a localisation error of 1 .'9. Afterwards, the position was revised, but remained consistent within 0.'5. The latest position available as of July 2014 on the UK Swift Science Data Centre ${ }^{2}$ is centred at coordinates RA, Dec. $(\mathrm{J} 2000)=11^{\mathrm{h}} 05^{\mathrm{m}} 38^{\mathrm{s}} .97,-40^{\circ} 41^{\prime} 02^{\prime \prime}$. 6 with an error of 1 1.9, and about $6^{\prime \prime}$ offset from the initial XRT position (Fig. 1). Our analysis of the XRT emission compared with the optical upper limit reveals that this was a dark GRB (see Appendix A.2), therefore we included it in our survey aimed at searching for the hosts of this class of bursts (Rossi et al. 2012). Afterwards, we imaged the field with the Gamma-Ray burst Optical and Near-infrared Detector (GROND; Greiner et al. 2008), a sevenchannel imager mounted on the $2.2 \mathrm{~m} \mathrm{MPG/ESO}$ telescope on La Silla (Chile). We found a bright extended source partly covered by the enhanced error circle. We re-examined the MOA $R$ band images and found no source at the new position except the extended source (which is just barely visible), but it is possible that the large seeing $\left(4^{\prime \prime}-5^{\prime \prime}\right)$ prevents us from distinguishing the afterglow from this object. This galaxy is also visible in the Palomar Digitized Sky Survey. We identify it as a host galaxy candidate of GRB 050219A (GRBH 050219A; see Fig. 1).

To obtain a more complete view of the spectral energy distribution (SED) of the host candidate we searched the UVOT images obtained during the day of the GRB trigger. The UVOT $v$-band host detection is similar in depth to the MOA upper limit, thus we consider it unlikely that the afterglow could dominate the host in the UVOT $v$-band. Additionally, we retrieved and analysed archival FORS2/R-band images (ESO/VLT) and Spitzer Space Telescope observations. The field was also covered by the Galaxy Evolution Explorer (GALEX) UV survey ${ }^{3}$. Furthermore, members of our team were awarded observing time to target the host candidate with the Herschel Space Observatory (Hunt et al. 2014). The summary of the observations is given in Table B.1.

\footnotetext{
2 See www.swift.ac.uk/xrt_positions. The webpage offers a brief introduction (but see also Evans et al. 2009).

3 The field was covered by the Wide-Field Infrared Survey Explorer (WISE) and the galaxy is detected in the W1-band $(3.4 \mu \mathrm{m})$ but with a lower significance than in the IRAC $3.6 \mu \mathrm{m}$ band. Therefore, we do not consider this detection here.
} 
Finally, on the 19th of April 2012 we obtained UV to nearIR (NIR) spectroscopic observations of the host galaxy candidate with the X-Shooter instrument (Vernet et al. 2011) mounted on the VLT on Paranal (ESO, Chile) ${ }^{4}$. The observations were performed at seeing $\sim 1^{\prime \prime}$ and airmass $\sim 1.1$. The slit was placed along the parallactic angle which was at $\sim 40$ degrees counterclockwise from the north, as shown in Fig. 1. They consisted of four exposures with an exposure time of $600 \mathrm{~s}$ each. They were obtained by nodding along the slit with an offset of $5^{\prime \prime}$ between exposures in a standard ABBA sequence. We used slit widths of 1.'0, 0.'9 and 0.9 for the UVB, VIS and NIR spectrograph arms, respectively, resulting in resolving powers of $R=\lambda / \Delta \lambda \approx 4400,7400$ and 5400 .

\section{Data analysis}

Swift/UVOT data were analysed using the standard analysis software distributed within FTOOLS, version 6.5.1. The source count rates were extracted within a $3^{\prime \prime}$ aperture. An aperture correction was estimated from selected nearby point sources in each exposure and applied to obtain the standard UVOT photometry calibrated for a $5^{\prime \prime}$ aperture.

GROND $g^{\prime} r^{\prime} i^{\prime} z^{\prime}$ and $J H K_{\mathrm{s}}$ images were reduced in a standard manner using PyRAF/IRAF (Tody 1993). The procedure is based on the pipeline written to reduce GROND data (Yoldaş et al. 2008; Krühler et al. 2008). Photometry was performed by using an aperture diameter of 7 ", that is 2.5 times the full width at half maximum (FWHM) of the stellar point spread function (PSF) in $K$-band (the largest value among the GROND filters). Within this aperture the measured flux flattened in a curve-ofgrowth analysis and therefore it is sufficiently large to include all of the galaxy flux. GROND optical bands were calibrated against the secondary photometric standards listed in Table B.2. Their magnitudes were derived from observations of SDSS fields. GROND NIR bands were calibrated against 2MASS field stars. The FORS2/R-band image was analysed in the same way but calibrated using a zeropoint given by ESO. In the following, we will not use the FORS2 photometry because the accuracy of the GROND photometric calibration is better than the one available for the FORS2/R band. UV, optical and NIR magnitudes were corrected for Galactic extinction using the interstellar extinction curve derived by Cardelli et al. (1989) and by assuming $E(B-V)=0.16 \mathrm{mag}$ (Schlegel et al. 1998) and a visual extinction to reddening ratio of $R_{V}=3.1$. The photometry on Spitzer and Herschel data were performed with an aperture larger than 9 arcsec. The method is described by Hunt et al. (2014).

The host galaxy candidate is detected in the UVOT $v$-band, all GROND optical/NIR bands, the FORS2/R-band, and with both Spitzer IRAC and MIPS. The galaxy is not detected in the UV (GALEX and UVOT) or in Herschel bands. The summary is given in Table B.1. The coordinates of the host galaxy candidate are RA, Dec $(\mathrm{J} 2000)=11^{\mathrm{h}} 05^{\mathrm{m}} 39^{\mathrm{s}} .07,-40^{\circ} 41^{\prime} 04^{\prime \prime} 6$. They are derived from the GROND $r^{\prime}$-band which have an astrometric precision of about 0.3 corresponding to the rms accuracy of the USNO-B1 catalogue (Monet et al. 2003). Given coordinates of the new enhanced XRT position (see above) are offset $\sim 2^{\prime \prime}$ from the galaxy centre.

We processed the X-Shooter spectra using version 2.0.0 of the data reduction pipeline (Goldoni et al. 2006; Modigliani et al. 2010), using the reduction technique developed for nodded observations. To flux-calibrate the spectrum we used the

\footnotetext{
4 The observations were conducted as a part of the Italian Guaranteed Time under program 089.A-0843(A) (PI: Piranomonte).
}

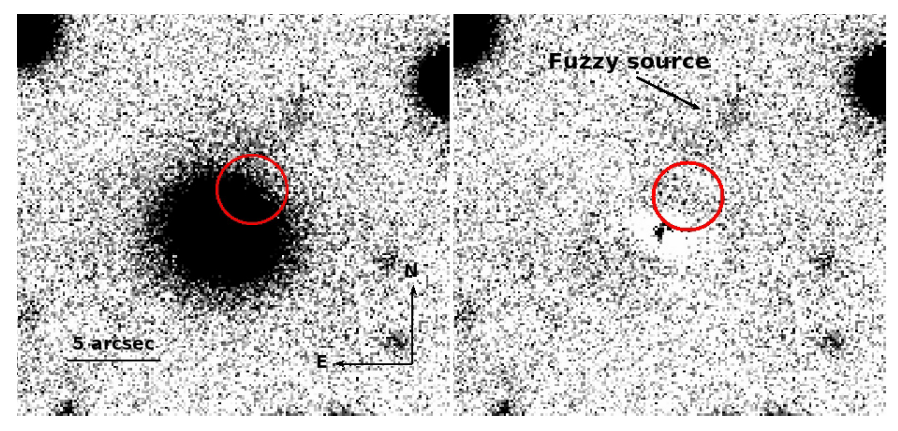

Fig. 2. Left: zoom into the FORS2/R-band image centred on the enhanced XRT error circle (in red) of GRB 050219A. Right: subtraction of the bright galaxy with GALFIT does not reveal any source within the XRT error circle. However, a fuzzy source is visible north-west and outside the XRT error circle (see Sect. 4.1 for details). The bright spot at the centre of the image is just a residual of the template subtraction (south-eastern border of the XRT circle).

observations of the spectrophotometric standard star LTT 3218, taken in the nodding mode.

Flux values were corrected for Galactic extinction. Afterwards, we cross-checked and calibrated the fluxes using the corresponding magnitudes of the galaxy (see Table B.1) and therefore accounting for slit-aperture flux losses, because the slit was much smaller than the entire galaxy. The final correction factor is 6.2 .

\section{Results}

\subsection{The host galaxy candidate and its morphology}

The host candidate has a featureless morphology with a FWHM of $\sim 1^{\prime \prime} .7$ both in the GROND $/ r^{\prime}$ and the FORS2/R-band images, i.e., larger than the stellar $F W H M\left(\sim 1^{\prime \prime}\right.$ for both observations), thus it is clearly a galaxy. The galaxy has a small elongation along the NE-SW axis $(a / b \sim 1.1$, where $a$ and $b$ are the semimajor and the semi-minor axis of the best matching ellipse) and an averaged half-light radius of $\sim 1^{\prime \prime} .3$.

No other close-by galaxies are visible in any of the images, and the few other objects within a few arcsec are stars or they are too faint to be clearly classified as galaxies. One of them is a fuzzy source barely visible in the FORS2/R-band image outside the north-west border of the XRT error circle and offset 4 ". 6 from its centre (Fig. 2). The source is detected at only $2 \sigma$ confidence level, thus it might not be real. If real it could be a galaxy, perhaps a small companion of the bright host candidate. Aperture photometry for this fuzzy source gives an extinction-corrected magnitude of $R \sim 25.5 \pm 0.6$, beyond the FORS2 $3 \sigma$ limitingmagnitude of $\sim 25.0$.

If there were another galaxy within the XRT error circle, the glare of the bright galaxy makes its identification very hard. Therefore, we used GALFIT v.3.0.5 (Peng et al. 2010) to subtract the bright galaxy (within the XRT error circle) in the FORS2/R-band image and search for possible hidden objects. We obtained the best solution using a 2D Sérsic profile with an index $n=3.3 \pm 0.2$ and an effective radius equal to its half-light radius (1". 3 ; see above). The profiles were convolved with the stellar PSF. To build the PSF we measured the magnitudes of isolated stars with ten different apertures with a radius in the interval $(F W H M, 2 \times F W H M)$. Afterwards, we fitted their profile using the psf routine under the DAOPHOT/IRAF package, following the prescriptions outlined in Stetson (1987) and in the DAOPHOT manual. The PSF image model to be used in GALFIT 


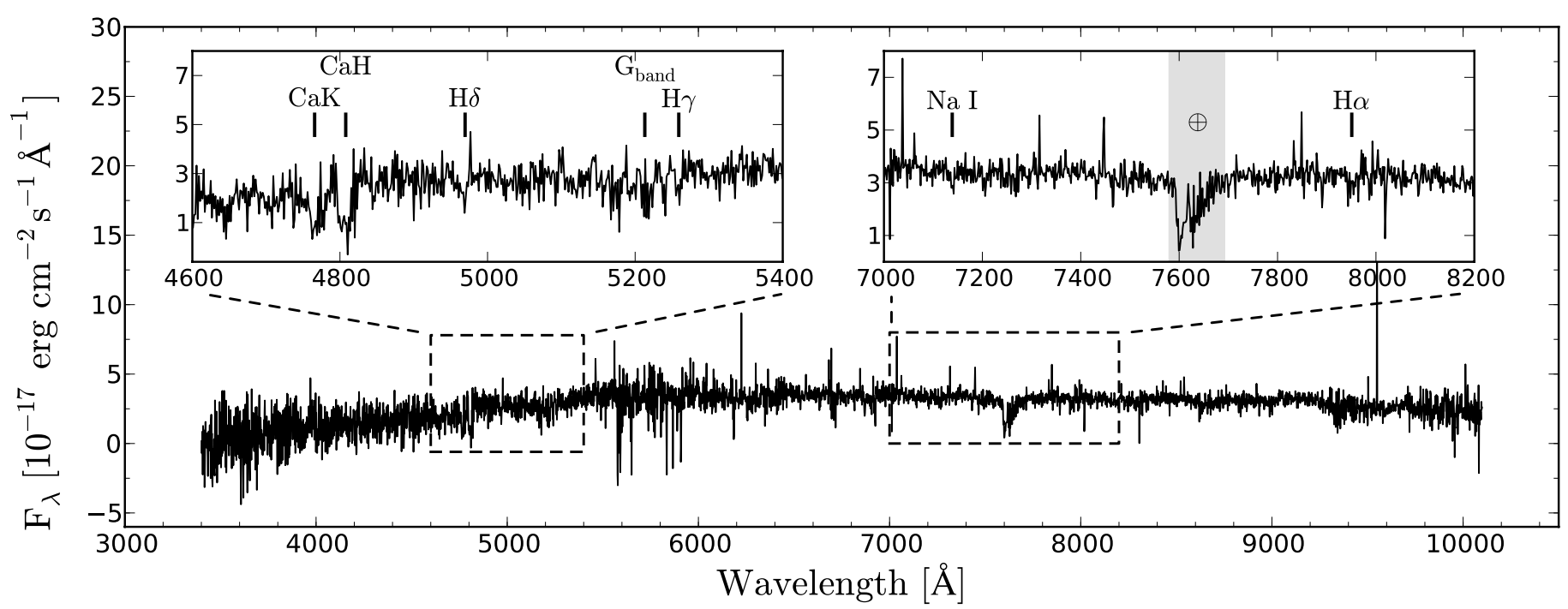

Fig. 3. X-Shooter (UVB and VIS arms) spectrum of the putative host galaxy of GRB 050219A (Fig. 1). Several absorption lines are detected at a common redshift of $z=0.211$. The zoom-in panels show the regions where the absorption features are visible. Only continuum is detected in the infrared X-Shooter arm, therefore it is not presented in the plot.

was obtained with the task seepsf and finally normalised. After subtraction, no sources are detected within the enhanced XRT error circle (right panel in Fig. 2).

Because of the featureless morphology and the best fit with a Sérsic profile having $n>2$, we classify the host candidate as an early-type galaxy (ETG; e.g., Glazebrook et al. 1995; Rowlands et al. 2012).

\subsection{Spectroscopic redshift and star formation}

In the X-Shooter spectrum, the galaxy continuum is well visible and there are clear detections of absorption lines typical of an elliptical galaxy with an old stellar population (Ca H\&K, G-band, and several Balmer absorption lines including $\mathrm{H} \alpha, \mathrm{H} \delta$, and $\mathrm{H} \gamma$ ) at redshift $z=0.211$ (see Fig. 3). At this redshift, 1 arcsec on the sky corresponds to $3.4 \mathrm{kpc}$, the distance modulus is $m-M=$ $40.07 \mathrm{mag}$, and the look-back time is $2.57 \mathrm{Gyr}(11.15 \mathrm{Gyr}$ after the Big Bang). The half-light radius of the galaxy ( $\left.\sim 1^{\prime \prime} .3\right)$ corresponds to $\sim 4 \mathrm{kpc}$ and its projected offset from the centre of the XRT position is $\sim 6 \mathrm{kpc}$. We find a $3 \sigma$ flux excess at the position of the [O II] emission line (Fig. 4). The spectrum does not allow us to resolve the [O II] doublet. This is due to the poor signal-tonoise ratio and the dispersion velocity of the gas in the galaxy that is comparable to the relative separation of the two lines of the [O II] doublet. We find a flux (calibrated and corrected for the aperture; Sect. 3) of $8.7 \times 10^{-17} \mathrm{erg} \mathrm{cm}^{-2} \mathrm{~s}^{-1}$, which corresponds to a SFR of $0.06_{-0.02}^{+0.01} M_{\odot} \mathrm{yr}^{-1}$ following the prescription given in Savaglio et al. (2009). Note that the slit aperture does not intersect the centre of the XRT error circle, where there could be additional star formation, signalled by the explosion of the long GRB. However, this star formation should be visible in the SED.

\subsection{Modelling of the spectrum}

We modelled the X-Shooter spectrum of the host candidate using the GANDALF software (Sarzi et al. 2006) to investigate the likelihood of the detection of the [O II] line. GANDALF linearly combines a set of stellar templates convolved with a line-ofsight velocity dispersion and fits galaxy spectra (Cappellari \& Emsellem 2004). At the same time it also fits a user-defined list of gas emission lines modelled with Gaussian templates. As stellar templates we used a subset of the X-Shooter Spectral Library (XSL; Chen et al. 2014). The input spectrum was flux-calibrated and de-reddened as described above. It was then rebinned logarithmically to have a constant velocity dispersion and fit between 3600 and $4100 \AA$ in the rest frame. This spectral region was chosen because of the presence of [O II] and of the strong $\mathrm{Ca}$ II H $\& \mathrm{~K}$ feature which helps in the convergence of the fit. We obtained a best fit stellar continuum with a combination of $\mathrm{G}, \mathrm{K}$ and $\mathrm{M}$ stars and the [O II] line was found in the best fit model with a flux of $7.1 \pm 2.2 \times 10^{-17} \mathrm{erg} \mathrm{cm}^{-2} \mathrm{~s}^{-1}$ (Fig. 4, bottom panel). No further extinction is necessary to fit the spectrum. We thus confirm the presence of the [O II] line at the $\sim 3 \sigma$ level.

$\mathrm{H} \alpha$ and $\mathrm{H} \beta$ emissions are not detected in our spectrum. Balmer lines are more uncertain than [O II] due to the undetermined underlying stellar absorption, which is more important in older stellar populations. The $\mathrm{H} \alpha, \mathrm{H} \beta$, and [O III] emission lines are constrained by GANDALF to $<7 \times 10^{-17} \mathrm{erg} \mathrm{cm}^{-2} \mathrm{~s}^{-1}$ at $3 \sigma$, therefore confirming their non-detection in the X-Shooter spectrum. The upper limit on $\mathrm{H} \alpha$ corresponds to a $S F R \lesssim$ $0.04 M_{\odot} \mathrm{yr}^{-1}$, consistent with the SFR determined with [OII]. The other upper limits are not constraining. However, we prefer to take the given upper limits with caution because $\mathrm{H} \alpha$ and $\mathrm{H} \beta$ emission lines are in a noisy part of the spectrum and fall within the corresponding absorbing lines, decreasing the chance of detection. Therefore, given our data set, the best SFR estimator is the $[\mathrm{O} \mathrm{II}]$ emission line.

The non-detection of the Balmer emission lines prevents us from constraining the extinction; however our analysis of the spectrum with GANDALF showed no evidence for extinction by dust. This is not surprising, because there are intriguing examples of dust-extinguished LGRBs hosted in blue and young galaxies with very low global extinction (e.g., Krühler et al. 2011). In general, the global dust extinction in GRBHs is in many cases negligible (e.g., Hunt et al. 2014). This is further supported by the low average extinction measured along GRB sight-lines $\left(A_{V}<0.4 \mathrm{mag}\right)$ and the fact that afterglows with negligible $A_{V}$ are common (e.g., Kann et al. 2010; Covino et al. 2013). Taken together, these cases illustrate that the starforming regions typically associated with LGRBs have patchy 

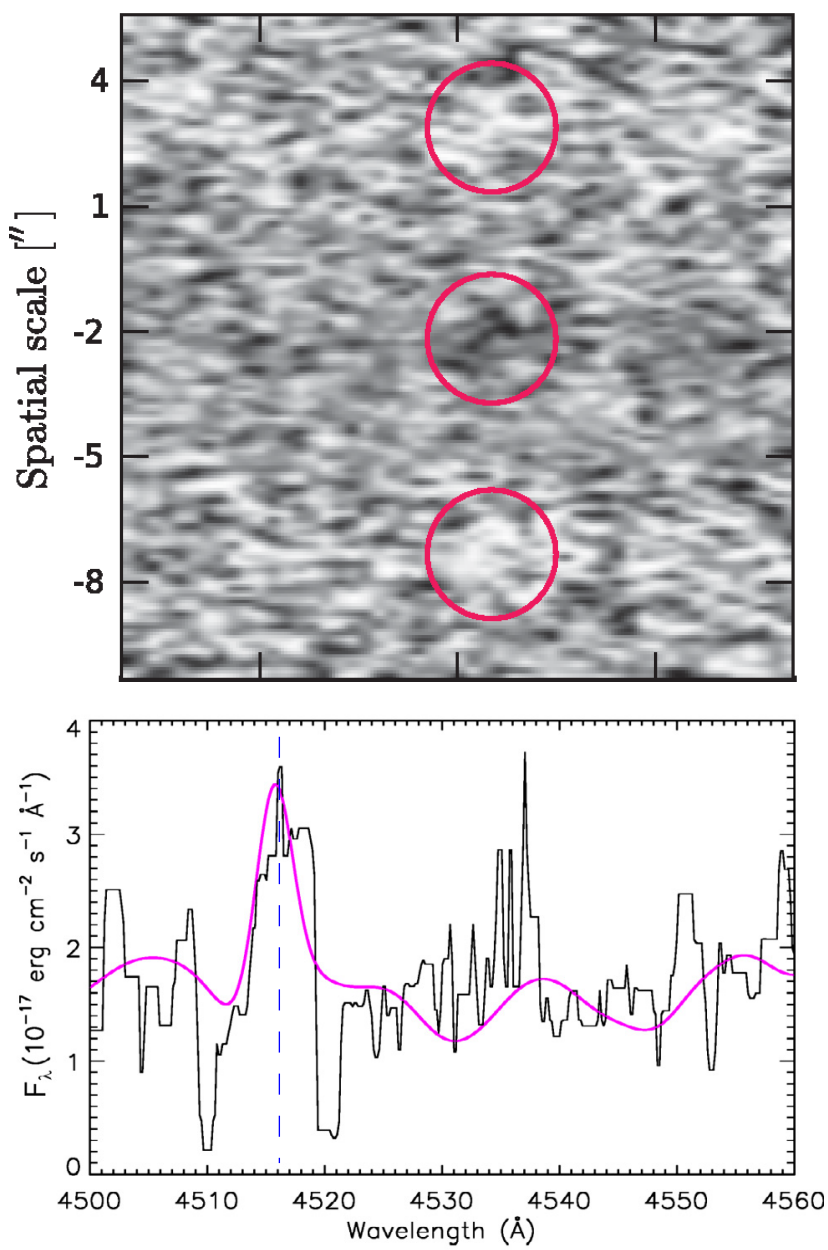

Fig. 4. Zoom-in of the region occupied by the [O II] emission line in the X-Shooter spectrum. Top: 2D spectrum. The circles mark the emission line and the negative features resulting from the nodding. Bottom: modeling of the spectrum obtained with GANDALF (magenta line) compared to the one-dimensional spectrum. The spectrum has been median filtered, flux-calibrated, and corrected for Galactic extinction. The [O II] emission line is redshifted to $4516 \AA$ (blue dashed line) in agreement with the redshift of 0.211 measured with the absorption lines only.

dust distributions. Therefore, the dust extinction depends on the geometry of the dust distribution, and not on the global properties of the host galaxy. However, note that even if we assume a reasonable $A_{V}=1 \mathrm{mag}$ in the star-forming region in agreement with the dark nature of GRB 050219A, the SFR would be just a factor of 2.5 higher.

\subsection{Modelling of the spectral energy distribution}

The SED of the host candidate of GRB 050219A has been modelled making use of template SEDs developed with $\mathrm{GraSil}^{5}$ (Silva et al. 1998) and using the SED-fitting procedure described in Lo Faro et al. (2013). Differently from semi-empirical approaches GraSil is a self-consistent physical model which allows the SEDs of galaxies to be predicted from the far-UV to radio including a state-of-the-art treatment of dust extinction and reprocessing based on a full radiative transfer solution. Moreover, it includes star-formation histories (SFHs) which are self-consistently computed following the chemical evolution of the galaxy. The photometric data of the host candidate are best-fit

\footnotetext{
5 www.adlibitum.oat.ts.astro.it/silva
}

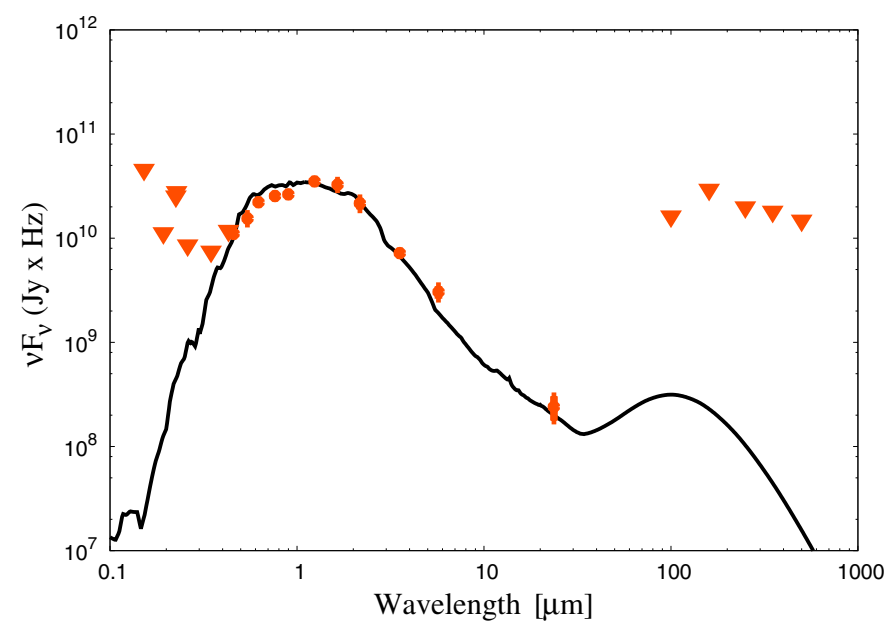

Fig. 5. SED fitting of the host galaxy candidate of GRB 050219A with GraSil from UV (GALEX) to sub-mm wavelengths (Herschel). The SED is best fit by a galaxy template with the following properties: age $\sim 3 \mathrm{Gyr}$, SFR $\lesssim 0.1 M_{\odot} \mathrm{yr}^{-1}$, and stellar mass $M_{*} \sim 10^{9.98} M_{\odot}$. The IR star formation and dust mass are not constrained by the observed Herschel upper limits.

by a galaxy with a 3 Gyr-old stellar population with a stellar mass of $10^{9.98} M_{\odot}$ and negligible dust content $A_{V}<0.1 \mathrm{mag}$ (Fig. 5). We also find the photometric data set to be consistent with a $S F R \lesssim 0.1 M_{\odot} \mathrm{yr}^{-1}$. These results are in good agreement with a previous SED fit presented by Hunt et al. (2014), who used an older set of SED templates and finds a stellar mass of $10^{9.91} M_{\odot}$. We conclude that the measurement of the [O II] line provides a good estimate of the current SFR. We noted in Sect. 4.2 that the X-Shooter slit does not intersect the centre of the XRT circle, and there could be additional star formation. However, our aperture photometry covers the whole galaxy and partly the XRT circle, and any star-forming region in the galaxy contributes to the observed SED, including those not covered by the X-Shooter slit. Therefore, the SED fitting provides an upper limit to the global SFR of the host candidate.

The parameters estimated via SED-fitting are notoriously affected by degeneracy and it is therefore difficult to estimate their errors. In particular, age and stellar masses tend to differ according to the adopted SFHs. The best fit template is based on a SFH with an infall time scale $\tau=0.1 \mathrm{Gyr}$ and an efficiency of 2.3, typical of galaxies dominated by an old stellar population (e.g., Silva et al. 1998). With this SFH, we have obtained a reasonable result in the age range of $3_{-0.5}^{+1}$ Gyr. A discussion of the derivation of galaxy properties and typical uncertainties is given by Michałowski et al. (2010, 2012a, 2014).

\subsection{Is there AGN activity?}

Since the $[\mathrm{O}$ II] emission line is also observed in active galactic nuclei (AGNs), one might wonder whether the line observed in the galaxy spectrum is due to nuclear activity. Former studies have shown that GRBHs do not generally show signatures of AGN activity in their emission lines (Watson et al. 2011) and in their SEDs (Michałowski et al. 2008). Note that [O II] alone is not sufficient to claim the presence of nuclear activity. but other lines are necessary. In particular, there is no [O III] emission visible in the spectrum, which in AGNs is usually stronger or at least comparable to the flux observed in [O II] (e.g., Kewley et al. 2007). X-ray emission is one of the principal characteristics of AGN activity. No X-ray source is known at the position 
of the bright host candidate or in the 10 arcmin surrounding radius. The Swift/XRT observations provide an upper limit of $\sim 1.7 \times 10^{-14} \mathrm{erg} \mathrm{cm}^{-2} \mathrm{~s}^{-1}$ (see the Swift/XRT on-line repository ${ }^{6}$ ). At $z=0.211$ this corresponds to a luminosity $L_{X}<$ $2.4 \times 10^{42} \mathrm{erg} \mathrm{s}^{-1}$, which is enough to constrain the presence of an AGN. However, note that AGN activity affects the mid-IR SED with a power-law component that is not detected in the observed SED (see Sect. 4.4). Therefore, the [O II] emission line is most likely due to on-going star formation rather than nuclear activity.

\section{Discussion}

\subsection{Comparison with apparently similar cases}

If GRB 050219A were a short GRB (e.g., Berger 2014), the association with a galaxy having a low SFR dominated by an old stellar population would not be unexpected; more than $50 \%$ of all short GRBHs have an evolved stellar population, and ETGs are common (e.g., Leibler \& Berger 2010; Berger 2011). However, the possibility that GRB 050219A is a short burst is excluded, because not only does it have a long duration $\left(T_{90} \sim 24 \mathrm{~s}\right)$ but it also satisfies the $E_{\mathrm{p}, \mathrm{i}}-E_{\mathrm{iso}}$ and the lag-luminosity correlations for LGRBs (Sect. A.1).

The case studied here is apparently similar to the LGRB 060912A (Levan et al. 2007) and the LGRB 130702A (Kelly et al. 2013). In the first case, the initial images showed that the galaxy closest to the GRB position was a low-redshift elliptical galaxy $10^{\prime \prime}$ away from the nominal locus. The situation changed when the deepest VLT imaging was acquired, showing that the real host is a star-forming galaxy at $z=0.937$ lying within the XRT error circle. In the case of the LGRB 130702A, the explosion site is offset $\sim 7$.' 6 from the centre of a bright red disk-dominated galaxy, but $\sim 0 . ' 6$ away from the centre of a much fainter metal-poor dwarf galaxy. Both galaxies have the same spectroscopic redshift as the GRB afterglow $(z=0.145)$ and form a gravitationally bound system. Kelly et al. (2013) concluded that the dwarf galaxy is the most likely host of LGRB 130702A on the basis of the smaller offset from the GRB site.

The case of GRB 050219A represents the converse situation: the bright ETG host candidate is the closest (in projection) to the XRT error circle and it partially overlaps with the circle. Therefore, the chance probability of a misidentification is much lower.

Kelly et al. (2013) argued that GRB 130702A is a lucky case where the red massive galaxy and its dwarf satellite (i.e., the GRB host) are well separated in the sky, but the same situation can result, in some cases, in a superposition along the sight line and in this case it would be a lot harder to distinguish the two galaxies. We have shown that no other candidates are detected even after image subtraction. In the following we will investigate the properties that a candidate host should have in order to not be detected in the case of GRB 050219A. If we take the dwarf host of GRB 130702A as a typical dwarf GRBH at low redshift we can calculate how it would appear at $z=0.211$. In order to keep the same absolute magnitude $\left(M_{r} \sim-16 \mathrm{mag}\right)$, it would have an apparent magnitude $r \sim 24.0 \mathrm{mag}$. Therefore, it would be clearly detected in the FORS2 image that has a $3 \sigma$ limiting magnitude of $R \sim 25 \mathrm{mag}$. To have the same $R$-band magnitude as the fuzzy object, the dwarf GRBH 130702A would be at $z \sim 0.45$. Of

\footnotetext{
6 http://www.swift.ac.uk/xrt_spectra/; Evans et al. (2007, 2009).
}

course a dwarf galaxy, like the putative companion of the bright host candidate can even be fainter than GRBH 130702A. We conclude that an alternative host candidate for 050219A should be redder and/or fainter than GRBH 130702A, or should lie at a redshift significantly higher than $z=0.211$.

\subsection{How likely is the association of the host candidate with GRB 050219A?}

GRB 050219A was selected from a parent sample of optically faint GRBs but with Swift/XRT detections. The details of selection criteria are explained in Rossi et al. (2012). About 40 targets fulfil these selection criteria. However, due to the limited amount of observing time the sample was restricted to 18 targets. We caution that the sample is not complete because it is biased towards dark GRBs and not representative of the whole GRBH population. It is therefore difficult to estimate the probability of a random association of dark GRBs with a particular class of galaxies like ETGs.

In general, the only way to unambiguously associate a host candidate with a GRB is to obtain the redshifts of afterglow and galaxy and find them to be identical. When no optical afterglow is seen, as is typical of dark GRBs, the host is chosen on the basis of positional coincidence and the chance probability (Bloom et al. 2002). It is also possible to do this by relying on the properties of the candidate host(s), including colour and SFR, in order to identify star-forming galaxies (e.g., Rossi et al. 2012).

Bloom et al. (2002) introduced a criterion to calculate the probability $p$ of finding a galaxy of given (extinction-corrected) $R$-band magnitude in a circular region of radius $r$. These authors discuss three possible scenarios for determining the radius: i) the GRB is well localised inside the detectable light of a galaxy; ii) the localisation is poor and the host candidate is within the error circle; iii) the localisation is good but it is outside the light of the nearest galaxy. The most appropriate scenario is the one that gives the largest radius. The case of the putative host of GRB 050219A is in between scenarios ii) and iii) and the radius is $r=3 . \prime 5$, given by the $3 \sigma$ confidence level of the XRT localisation error. Following Bloom et al. (2002), we used the $R$-band counts of galaxies brighter than 27 mag of a field at high galactic latitude presented by Hogg et al. (1997). We find that the chance probability ( $p$-value) that a galaxy with an equivalent (or brighter) magnitude $(R \sim 20)$ lies within a 3". 5 radius of the XRT position is $\sim 0.8 \%$. For the fuzzy source possibly detected in the FORS2 image with $R \sim 25.5$, the radius is given by the third scenario described above. Following Bloom et al. (2002), the radius is given by $\sqrt{r_{\text {of }}^{2}+r_{\text {half }}^{2}}=5^{\prime \prime}$, where $r_{\text {of }}$ is the offset of the centre of the source from the XRT position (4.' 6 ) and $r_{\text {half }}$ is the half-light radius of the source $\left(\sim 1^{\prime \prime}\right)$. For the fuzzy source we find a $p$-value of $\sim 68 \%$.

We conclude that the small chance probability for the bright candidate and the high probability for the fuzzy object supports the idea that the bright galaxy in the XRT error circle is the best host candidate. and in the following we will simply refer to it as the host galaxy candidate, or GRBH 050219A.

\subsection{The host galaxy candidate of GRB 050219A compared with general galaxy populations}

It is justified to assume that the progenitor of the LGRB 050219A was a massive star which exploded as a broad-line Type-Ib/c supernovae (SN Ic-BL, associated with LGRBs, e.g., Bersier 2012; Schulze et al. 2014). Recently, 

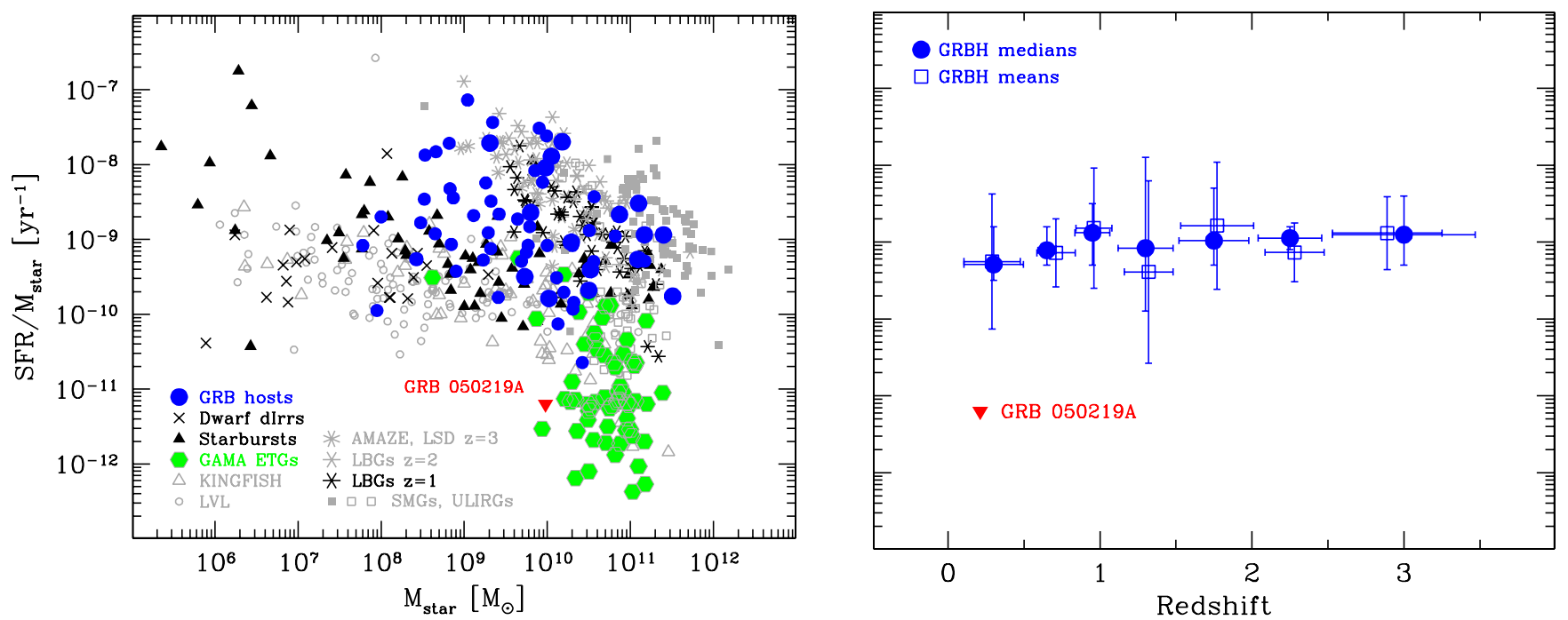

Fig. 6. Host galaxy of GRB 050219A compared to other GRBHs and other populations of galaxies (adapted from Hunt et al. 2014). Left: sSFR vs. stellar mass. The host of GRB 050219A (marked in red) lies in a region occupied by ETGs from the GAMA survey (Rowlands et al. 2012, marked in green). The best-studied GRBHs are marked in blue. Right: sSFR plotted against redshift for the GRBH sample from Hunt et al. (2014). The filled circles show the medians of each redshift bin, and the vertical error bars correspond to the upper and lower quartiles of the GRBH distributions. The empty squares show the mean of the GRBH distributions within each redshift bin, and the error bars correspond to the standard deviation. The horizontal error bars are the width of the redshift bins (for the GRBH data).

Table 1. Summary of the spectral properties of GRBH 050219A and other post-starburst galaxies.

\begin{tabular}{lccccccc}
\hline \hline Galaxy & Redshift & $\begin{array}{c}E W(\mathrm{H} \delta) \\
(\AA)\end{array}$ & $\begin{array}{c}E W[\mathrm{O} \text { II }] \\
(\AA)\end{array}$ & $\log \left(M_{*} / M_{\odot}\right)$ & $\begin{array}{c}g^{\prime}-r^{\prime} \\
\mathrm{mag}\end{array}$ & $\begin{array}{c}\mathrm{SFR}([\mathrm{OII}]) \\
\left(M_{\odot} \mathrm{yr}^{-1}\right)\end{array}$ & $\begin{array}{c}M_{*} / \mathrm{SFR} \\
(\mathrm{Gyr})\end{array}$ \\
\hline GRBH 050219A & 0.211 & 2.01 & -3.17 & 9.98 & 0.93 & 0.06 & 170 \\
GDDS-12-8139 & 1.189 & 8.36 & -6.21 & 10.39 & 0.40 & 2.3 & 10.5 \\
GDDS-02-0715 & 1.133 & 3.01 & -4.29 & 11.25 & 0.37 & 1.8 & 101.6 \\
GDDS-02-1543 & 1.131 & 4.22 & -5.34 & 10.80 & 0.67 & 0.2 & 267.4 \\
GDDS-12-8983 & 0.963 & 0.72 & -1.51 & 10.70 & 0.56 & 0.6 & 89.7 \\
\hline
\end{tabular}

Notes. The post-starburst galaxies are taken from Le Borgne et al. (2006).

Kelly et al. (2014) compared hosts of LGRBs and SNe Ic-BL to those of core-collapse SNe from the SDSS survey (Ahn et al. 2014). Compared to Kelly et al. (2014, their Fig. 10) GRBH 050219A has a lower sSFR, and with a half-light radius of $\sim 4 \mathrm{kpc}$ (see Sect. 4) it is larger than GRBHs and galaxies hosting SNe Ic-BL which usually have a radius $r \lesssim 2 \mathrm{kpc}$ (Kelly et al. 2014, their Fig. 8). However, the host of SN 2010ah has a similarly low SFR $\left(S F R \sim 0.1 M_{\odot} \mathrm{yr}^{-1}\right)$ and radius $(\sim 4.4 \mathrm{kpc})$.

Hunt et al. (2014) present new Herschel/far-IR data for 17 host galaxies, where 14 of them are hosts of dark GRBs. These galaxies are then included in a larger sample of 66 hosts combining those presented by Savaglio et al. (2009) and Perley et al. (2013). In Fig. 6 we show the sSFR vs. stellar mass and redshift from Hunt et al. (2014) and highlight GRBH 050219A. In particular the right panel shows mean and median of the sSFR for each redshift bin of the GRBH distribution, which are consistent within the errors. GRBH 050219A stands out as an isolated case because it has the lowest specific SFR among all the known hosts to date $\left(s S F R \lesssim 10^{-11} \mathrm{yr}^{-1}\right.$, using the result from the SED fitting). This low sSFR corresponds to UV brightness at $0.2 \mu \mathrm{m}$ of $\gtrsim 24 \mathrm{mag}$, in agreement with the GALEX upper limits. Using the distance modulus at $z=0.211(\mathrm{~m}-M=$ 40.07) and the photometry in Table B.1 we obtain the colours $M(N U V)-M(r) \gtrsim 4.5$ and $M(r)-M(J) \sim 1$. Galaxies with such red colours and low SSFR are usually called passive or quiescent galaxies to distinguish them from star-forming galaxies, and are consistent with an ETG population selected morphologically (e.g., Ilbert et al. 2010, 2013). Indeed, GRBH 050219A lies in a region occupied by galaxies morphologically classified as ETGs (Rowlands et al. 2012; left panel of Fig. 6). ETGs and especially elliptical galaxies are commonly associated with an old stellar population, and believed to be cold-gas and dust free. However, in the past years this picture has started to change due to the discovery that many ETGs contain significant amounts of gas and dust, and are actively forming stars, some with SFRs comparable to typical late-type spiral galaxies (e.g., Fukugita et al. 2004; Serra et al. 2012; Rowlands et al. 2012).

The spectrum of GRBH 050219A shows a $4000 \AA$ break as well as a strong $\mathrm{H} \delta$ absorption line coupled with little [O II] emission. These features are typical of the $\mathrm{H} \delta$-strong galaxy population (e.g., Dressler \& Gunn 1983; Balogh et al. 1999; Le Borgne et al. 2006). They are post-starburst galaxies, i.e., they have undergone a recent break in their star-formation activity and are transiting to a quiescent phase. They were more common at redshift $\sim 1$ and have decreased to a few percent today. In Table 1 we summarise the spectral features of GRBH 050219A together with those of some post-starburst galaxies studied by Le Borgne et al. (2006). The host galaxy candidate of GRB 050219A might be a low-redshift and low-mass member of this galaxy population. Although GRBH 050219A is less massive, its spectrum is otherwise similar to the poststarburst galaxy GDDS-12-8139 (Fig. 7). The relatively strong 


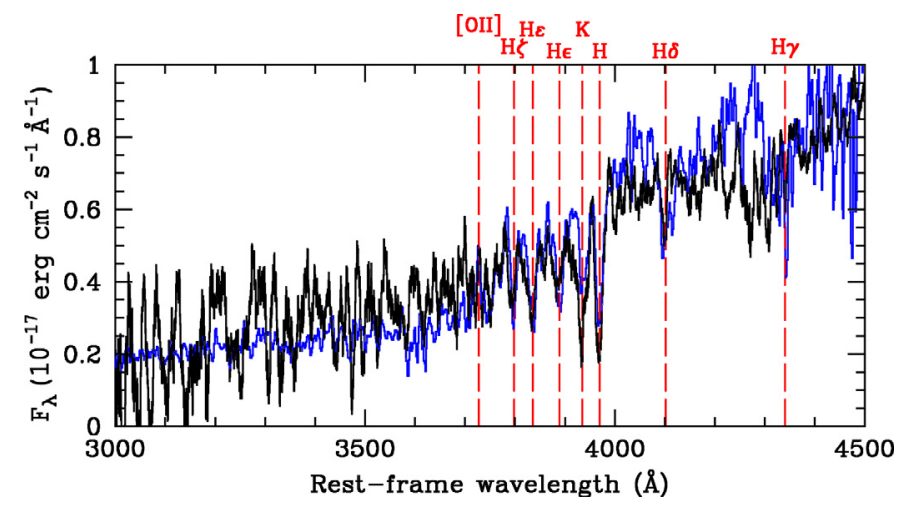

Fig. 7. X-Shooter spectrum of GRBH 050219A (black) compared with the spectrum of the post-starburst galaxy GDDS-12-8139 (Le Borgne et al. 2006). We marked the positions of the [O II] emission line as well as the most visible absorption lines.

$\mathrm{H} \delta$ line and the presence of [O II] emission could be the echoes of intense episodes of star formation that faded 500 Myr prior to the epoch of observation. An alternative explanation for the recent star formation in post-starburst galaxies is the interaction of an old galaxy with a companion (e.g., Zabludoff et al. 1996; Goto 2005). This can also be the case for GRBH 050219A, which possibly interacted in the past with the close (in projection) fuzzy source. We cannot prove this interaction, but we speculate that the small amount of star formation responsible for the weak [O II] emission and the explosion of the burst may be the sign of interaction between the quiescent galaxy and a possible companion.

Core-collapse SNe are extremely rare in ETGs but not impossible. For example, Kawabata et al. (2010) discovered the Type-Ib SN $2005 \mathrm{cz}$ in the local elliptical galaxy NGC 4589. Also, Perets et al. (2010) report that SN 2005E is hosted by an elliptical galaxy that also includes a small and young stellar population with life times of $10^{7}-10^{8}$ years, consistent with the core-collapse model. Hakobyan et al. (2012) found only four core-collapse SNe in ETGs in a sample of 2104 SN host galaxies (see also Hakobyan 2008; Leaman et al. 2011). Since these events are related to star formation, their host galaxies could be interacting galaxies, where a starburst was triggered by galaxygalaxy interaction (e.g., Fruchter et al. 1999; Wainwright et al. 2007; Chen 2012).

\section{Summary}

We demonstrated that GRB 050219A is a rightful member of the LGRB population. Within its XRT error circle we found a relatively bright galaxy, detected from the optical to the mid-IR bands. Its morphology and the absorption lines in the $\mathrm{X}$-Shooter spectrum suggest that this is an ETG dominated by an old stellar population at redshift $z=0.211$. We identified this galaxy as the most probable host galaxy of GRB 050219A on the basis of its low chance probability. It has the lowest SSFR among all known LGRB hosts $\left(s S F R=S F R / M_{*} \lesssim 6 \times 10^{-12} \mathrm{yr}^{-1}\right)$, but is similar to other ETGs. The properties of GRBH 050219A, and in particular its size and sSFR, are on average different from those of other hosts of core-collapse SNe and LGRBs. The presence of strong stellar absorption features coupled with a weak [O II] emission line in the X-Shooter spectrum suggest that the host galaxy candidate is a low-mass member of the post-starburst galaxy population. We concluded that it is the first quiescent ETG and the first post-starburst galaxy found to probably host a LGRB. Additional observations are required in order to exclude alternative host galaxies like the faint fuzzy source outside the XRT circle, and to study the high-mass star formation at the burst location.

Several years ago the host galaxies of LGRBs were believed to be sub-luminous, blue compact dwarfs. In the past years this view has changed thanks to the discovery of massive and dusty hosts of dark LGRBs (e.g., Hunt et al. 2011; Rossi et al. 2012; Perley et al. 2013). The discovery of a LGRB 050219A in an quiescent ETG would be further evidence that LGRBs can explode in a variety of galaxies with the only requirement being an episode of high-mass star formation.

Acknowledgements. We are grateful to the anonymous referee for constructive comments that improved the clarity of the manuscript. A.R., E.P. and L.K.H. acknowledge support from PRIN-INAF 2012/13. SP acknowledges partial support from PRIN MIUR 2009 and from ASI INAF I/004/11/1. A.R. and D.A.K. acknowledge support by the Thüringer Landessternwarte Tautenburg. M.J.M. acknowledges the support of the Science and Technology Facilities Council. A.N.G. acknowledges support by DFG grant Kl 766/16-1. S. Schulze acknowledges support from CONICYT through FONDECYT grant 3140534, from Basal-CATA PFB-06/2007, Iniciativa Cientifica Milenio grant P10-064-F (Millennium Center for Supernova Science), by Project IC120009 "Millennium Institute of Astrophysics (MAS)" of Iniciativa Científica Milenio del Ministerio de Economiía, Fomento y Turismo de Chile, with input from "Fondo de Innovación para la Competitividad, del Ministerio de Economía, Fomento y Turismo de Chile". P.S. acknowledges support through the Sofja Kovalevskaja Award from the Alexander von Humboldt Foundation of Germany. E. Pian acknowledges partial support from contracts ASI INAF I/088/06/0, INAF PRIN 2011 and PRIN MIUR 2010/2011. Part of the funding for GROND (both hardware as well as personnel) was generously granted from the Leibniz-Prize to Prof. G. Hasinger (DFG grant HA 1850/28-1). We thank D. Malesani and P. M. Vreeswijk for their contribution to the analysis of the X-Shooter spectrum, and N. Masetti for insightful suggestions on many aspects of this study. This research has made use of the NASA/IPAC Extragalactic Database (NED) which is operated by the Jet Propulsion Laboratory, California Institute of Technology, under contract with the National Aeronautics and Space Administration. This work made use of data supplied by the UK Swift Science Data Centre at the University of Leicester.

\section{References}

Ahn, C. P., Alexandroff, R., Allende Prieto, C., et al. 2014, ApJS, 211, 17 Amati, L. 2006, MNRAS, 372, 233

Balogh, M. L., Morris, S. L., Yee, H. K. C., Carlberg, R. G., \& Ellingson, E. 1999, ApJ, 527, 54

Barthelmy, S. D., Barbier, L. M., Cummings, J. R., et al. 2005a, Space Sci. Rev., 120,143

Barthelmy, S. D., Chincarini, G., Burrows, D. N., et al. 2005b, Nature, 438, 994 Berger, E. 2011, New Astron. Rev., 55, 1

Berger, E. 2014, ARA\&A, 52, 43

Berger, E., \& Gonzalez, S. 2005, GCN Circ., 3048

Bersier, D. 2012, in Gamma-Ray Bursts 2012 Conf., Munich, PoS(GRB 2012)095

Bloom, J. S., Kulkarni, S. R., \& Djorgovski, S. G. 2002, AJ, 123, 111

Burrows, D. N., Hill, J. E., Nousek, J. A., et al. 2005, Space Sci. Rev., 120, 165 Cappellari, M., \& Emsellem, E. 2004, PASP, 116, 138

Cardelli, J. A., Clayton, G. C., \& Mathis, J. S. 1989, ApJ, 345, 245

Chabrier, G. 2003, PASP, 115, 763

Chen, H.-W. 2012, MNRAS, 419, 3039

Chen, Y.-P., Trager, S. C., Peletier, R. F., et al. 2014, A\&A, 565, A117 Covino, S., Melandri, A., Salvaterra, R., et al. 2013, MNRAS, 432, 1231

de Ugarte Postigo, A., Eguchi, S., Gorosabel, J., Yock, P., \& Castro-Tirado, A. J. 2005, GCN Circ., 3041

Dressler, A., \& Gunn, J. E. 1983, ApJ, 270, 7

Elliott, J., Greiner, J., Khochfar, S., et al. 2012, A\&A, 539, A113

Evans, P. A., Beardmore, A. P., Page, K. L., et al. 2007, A\&A, 469, 379

Evans, P. A., Beardmore, A. P., Page, K. L., et al. 2009, MNRAS, 397, 1177

Fruchter, A. S., Thorsett, S. E., Metzger, M. R., et al. 1999, ApJ, 519, L13

Fukugita, M., Nakamura, O., Turner, E. L., Helmboldt, J., \& Nichol, R. C. 2004, ApJ, 601, L127

Gehrels, N., Chincarini, G., Giommi, P., et al. 2004, ApJ, 611, 1005

Gehrels, N., Norris, J. P., Barthelmy, S. D., et al. 2006, Nature, 444, 1044

Glazebrook, K., Ellis, R., Santiago, B., \& Griffiths, R. 1995, MNRAS, 275, L19

Goad, M. R., Tyler, L. G., Beardmore, A. P., et al. 2007, A\&A, 476, 1401 
Goldoni, P., Royer, F., François, P., et al. 2006, in SPIE Conf. Ser., 6269 Goto, T. 2005, MNRAS, 357, 937

Graham, J. F., \& Fruchter, A. S. 2013, ApJ, 774, 119

Greiner, J., Bornemann, W., Clemens, C., et al. 2008, PASP, 120, 405

Greiner, J., Krühler, T., Klose, S., et al. 2011, A\&A, 526, A30

Hakobyan, A. A. 2008, Astrophysics, 51, 69

Hakobyan, A. A., Adibekyan, V. Z., Aramyan, L. S., et al. 2012, A\&A, 544, A81

Hjorth, J., \& Bloom, J. S. 2012, The Gamma-Ray Burst - Supernova Connection, 169

Hjorth, J., Sollerman, J., Møller, P., et al. 2003, Nature, 423, 847

Hjorth, J., Malesani, D., Jakobsson, P., et al. 2012, ApJ, 756, 187

Hogg, D. W., Pahre, M. A., McCarthy, J. K., et al. 1997, MNRAS, 288, 404

Hullinger, D., Barthelmy, S., Barbier, L., et al. 2005, GCN Circ., 3038

Hunt, L., Palazzi, E., Rossi, A., et al. 2011, ApJ, 736, L36

Hunt, L. K., Palazzi, E., Michałowski, M. J., et al. 2014, A\&A, 565, A112

Ilbert, O., Salvato, M., Le Floc'h, E., et al. 2010, ApJ, 709, 644

Ilbert, O., McCracken, H. J., Le Fèvre, O., et al. 2013, A\&A, 556, A55

Jakobsson, P., Hjorth, J., Malesani, D., et al. 2012, ApJ, 752, 62

Jakobsson, P., Hjorth, J., Fynbo, J. P. U., et al. 2004, ApJ, 617, L21

Kann, D. A., Klose, S., Zhang, B., et al. 2010, ApJ, 720, 1513

Karim, A., Schinnerer, E., Martínez-Sansigre, A., et al. 2011, ApJ, 730, 61

Kawabata, K. S., Maeda, K., Nomoto, K., et al. 2010, Nature, 465, 326

Kelly, P. L., Filippenko, A. V., Fox, O. D., Zheng, W., \& Clubb, K. I. 2013, ApJ, 775, L5

Kelly, P. L., Filippenko, A. V., Modjaz, M., \& Kocevski, D. 2014, ApJ, 789, 23

Kewley, L. J., Brown, W. R., Geller, M. J., Kenyon, S. J., \& Kurtz, M. J. 2007, AJ, 133,882

Kouveliotou, C., Meegan, C. A., Fishman, G. J., et al. 1993, ApJ, 413, L101

Krühler, T., Küpcü Yoldaş, A., Greiner, J., et al. 2008, ApJ, 685, 376

Krühler, T., Greiner, J., Schady, P., et al. 2011, A\&A, 534, A108

Le Borgne, D., Abraham, R., Daniel, K., et al. 2006, ApJ, 642, 48

Leaman, J., Li, W., Chornock, R., \& Filippenko, A. V. 2011, MNRAS, 412, 1419

Leibler, C. N., \& Berger, E. 2010, ApJ, 725, 1202

Levan, A. J., Jakobsson, P., Hurkett, C., et al. 2007, MNRAS, 378, 1439

Lo Faro, B., Franceschini, A., Vaccari, M., et al. 2013, ApJ, 762, 108

Michałowski, M. J., Hjorth, J., Castro Cerón, J. M., \& Watson, D. 2008, ApJ, 672,817
Michałowski, M., Hjorth, J., \& Watson, D. 2010, A\&A, 514, A67

Michałowski, M. J., Dunlop, J. S., Cirasuolo, M., et al. 2012a, A\&A, 541, A85

Michałowski, M. J., Kamble, A., Hjorth, J., et al. 2012b, ApJ, 755, 85

Michałowski, M. J., Hayward, C. C., Dunlop, J. S., et al. 2014, A\&A, 571, A75

Modigliani, A., Goldoni, P., Royer, F., et al. 2010, in SPIE Conf. Ser., 7737, 28

Monet, D. G., Levine, S. E., Canzian, B., et al. 2003, AJ, 125, 984

Peng, C. Y., Ho, L. C., Impey, C. D., \& Rix, H.-W. 2010, AJ, 139, 2097

Perets, H. B., Gal-Yam, A., Mazzali, P. A., et al. 2010, Nature, 465, 322

Perley, D. A., Levan, A. J., Tanvir, N. R., et al. 2013, ApJ, 778, 128

Romano, P., Perri, M., Beardmore, A., et al. 2005, GCN Circ., 3036, 1

Roming, P. W. A., Kennedy, T. E., Mason, K. O., et al. 2005, Space Sci. Rev., 120,95

Rossi, A., Klose, S., Ferrero, P., et al. 2012, A\&A, 545, A77

Rowlands, K., Dunne, L., Maddox, S., et al. 2012, MNRAS, 419, 2545

Sari, R., Piran, T., \& Narayan, R. 1998, ApJ, 497, L17

Sarzi, M., Falcón-Barroso, J., Davies, R. L., et al. 2006, MNRAS, 366, 1151

Savaglio, S., Glazebrook, K., \& Le Borgne, D. 2009, ApJ, 691, 182

Savaglio, S., Rau, A., Greiner, J., et al. 2012, MNRAS, 420, 627

Schlegel, D. J., Finkbeiner, D. P., \& Davis, M. 1998, ApJ, 500, 525

Schulze, S., Malesani, D., Cucchiara, A., et al. 2014, A\&A, 566, A102

Serra, P., Oosterloo, T., Morganti, R., et al. 2012, MNRAS, 422, 1835

Silva, L., Granato, G. L., Bressan, A., \& Danese, L. 1998, ApJ, 509, 103

Spergel, D. N., Verde, L., Peiris, H. V., et al. 2003, ApJS, 148, 175

Stetson, P. B. 1987, PASP, 99, 191

Tagliaferri, G., Goad, M., Chincarini, G., et al. 2005, Nature, 436, 985

Tody, D. 1993, in Astronomical Data Analysis Software and Systems II, eds. R. J. Hanisch, R. J. V. Brissenden, \& J. Barnes, ASP Conf. Ser., 52, 173

Ukwatta, T. N., Stamatikos, M., Dhuga, K. S., et al. 2010, ApJ, 711, 1073

van der Horst, A. J., Kouveliotou, C., Gehrels, N., et al. 2009, ApJ, 699, 1087

Vernet, J., Dekker, H., D’Odorico, S., et al. 2011, A\&A, 536, A105

Wainwright, C., Berger, E., \& Penprase, B. E. 2007, ApJ, 657, 367

Watson, D., French, J., Christensen, L., et al. 2011, ApJ, 741, 58

Woosley, S. E., \& Bloom, J. S. 2006, ARA\&A, 44, 507

Yoldaş, A. K., Krühler, T., Greiner, J., et al. 2008, in AIP Conf. Ser. 1000, eds. M. Galassi, D. Palmer, \& E. Fenimore, 227

Zabludoff, A. I., Zaritsky, D., Lin, H., et al. 1996, ApJ, 466, 104

Zhang, B., Zhang, B.-B., Virgili, F. J., et al. 2009, ApJ, 703, 1696

Pages 10 to 12 are available in the electronic edition of the journal at http://www . aanda.org 


\section{Appendix A: Properties of the GRB and its afterglow}

\section{A.1. Properties of the prompt emission}

In the last years, the detection of soft tails in short GRBs (e.g., GRB 050724, Barthelmy et al. 2005b) and of peculiar long events like GRB 060614 (Gehrels et al. 2006), have shown the limitations of the standard short/long classification based on duration only (e.g., Zhang et al. 2009). Thus, to test the nature of GRB 050219A and whether it is a real LGRB, we investigate whether this burst satisfies the $E_{\mathrm{p}, \mathrm{i}}-E_{\mathrm{iso}}$ correlation (Amati 2006). We based our analysis on the spectral fits, light curves and fluences reported in Tagliaferri et al. (2005) and on our personal analysis of the Swift/BAT spectrum. They show that the $\sim 24$ s pulse observed by Swift/BAT was followed by softer emission observed with the XRT, and thus the central engine was active for at least $\sim 120 \mathrm{~s}$. However, this later softer phase accounts only for $<20 \%$ of the total prompt emission and therefore the following conclusions do not depend on the softer emission. Luckily, the spectral peak falls within the BAT band and it is $E_{\mathrm{p}, \mathrm{i}}=90 \pm 9 \mathrm{keV}$. We find that assuming a redshift $z=0.211$ this burst is consistent with the $E_{\mathrm{p}, \mathrm{i}}-E_{\mathrm{iso}}$ correlation within $2 \sigma$. Any value between $0.2 \lesssim z \lesssim 2$ is good within $2 \sigma$. The GRB would lie in the region occupied by short GRBs only for $z \lesssim 0.1$ (see Fig. A.1).

We also checked the lag-luminosity relation, following the method described in Ukwatta et al. (2010). We assumed the same redshift as above, and we measure a peak luminosity of $(6.7 \pm 0.8) \times 10^{49} \mathrm{erg} \mathrm{s}^{-1}$. We find a lag of $3.4 \pm 1.2 \mathrm{~s}$ between BAT channels $3(50-100 \mathrm{keV})$ and $1(15-25 \mathrm{keV})$ and of $1.4 \pm 0.6 \mathrm{~s}$ between BAT channels $4(100-200 \mathrm{keV})$ and 2 (25$50 \mathrm{keV})$. Figure A.2 shows that GRB 050219A has one of the largest lags measured for a LGRB and it is in very good agreement with the lag-luminosity relation for LGRBs presented in Ukwatta et al. (2010).

Therefore, even though a redshift of about $0.5-1$ would place it in the regions of the $E_{\mathrm{p}, \mathrm{i}}-E_{\mathrm{iso}}$ and lag - luminosity planes most populated by typical LGRBs (see Figs. A.1 and A.2), the energetics, luminosity, spectrum and timing properties of GRB 050219A are consistent with the hypothesis that it is a LGRB at the redshift of the putative host galaxy.

\section{A.2. GRB 050219A was a dark burst}

Within the standard afterglow theory (Sari et al. 1998) the X-ray afterglow should have an X-ray spectral slope $\beta_{\mathrm{X}} \gtrsim 0.5$, and $\beta_{\mathrm{X}} \gtrsim 1$ in case of a break below the $\mathrm{X}$-ray frequency which is usually the case (Greiner et al. 2011). Jakobsson et al. (2004) quantify the optical dimness by testing whether the optical to $\mathrm{X}$-ray spectral slope $\beta_{\mathrm{OX}}$ is lower than 0.5 . We downloaded and analysed the XRT spectrum (both pc and wt modes) and we found $0.6<\beta_{\mathrm{X}}<1.2$, consistent with the values in the Swift/XRT on-line repository, but not good enough to distinguish between the two scenarios. However, without a break between optical and X-rays (i.e., $\beta_{\mathrm{OX}} \gtrsim 1$ ), the afterglow would have had $R \gtrsim 18$ at the time of the MOA observations, surely dominating over the galaxy light and well-detected, but we do not see this in

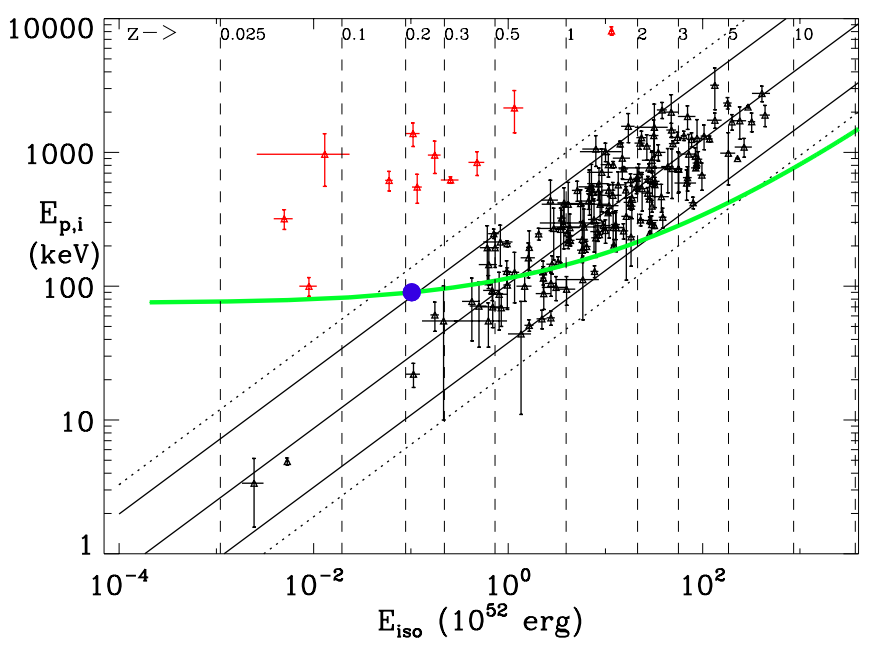

Fig. A.1. LGRBs (black) and short GRBs (red) in the $E_{\mathrm{p}, \mathrm{i}}-E_{\mathrm{iso}}$ plane. The blue dot indicates the position of GRB 050219A assuming $z=0.211$. The dashed vertical lines indicate the position of the GRB at different redshifts. The green line shows how this position changes together with the redshift. The solid and dotted lines show the $2 \sigma$ and $3 \sigma$ regions respectively.

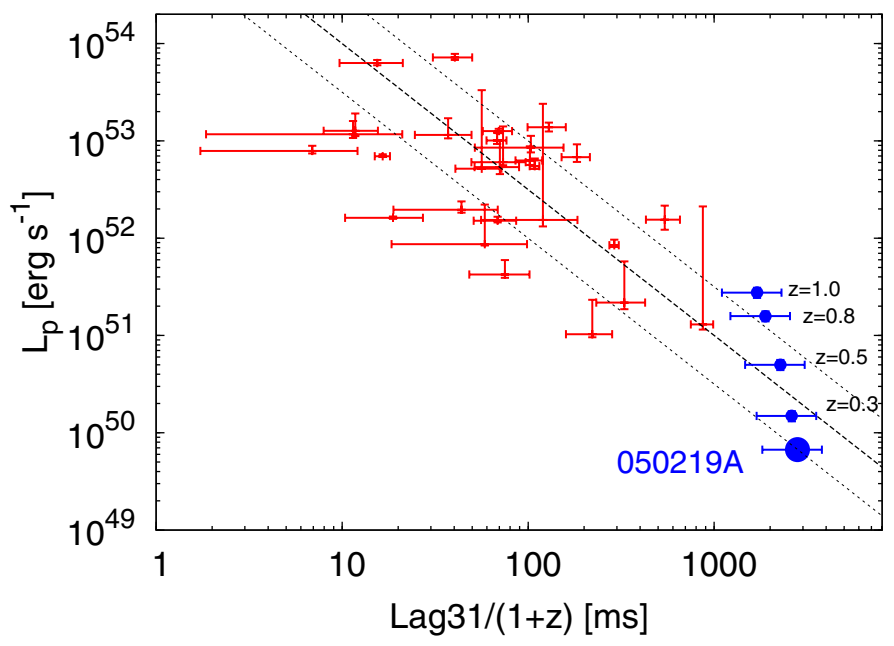

Fig. A.2. Isotropic peak luminosity as a function of spectral lag between BAT channels $3(50-100 \mathrm{keV})$ and $1(15-25 \mathrm{keV})$. The image is adapted from Ukwatta et al. (2010) and GRBs are marked in red. The dotted lines indicate the estimated $1 \sigma$ confidence level. We marked in blue the position of GRB 050219A at different redshifts, including the redshift of the bright galaxy at $z=0.211$ (bigger blue dot).

our re-analysis of the data. In the case of a break between optical and X-rays, lying close to the X-ray band, the afterglow should have been $R \sim 20$ or brighter. This is similar to the MOA upper limit, but because of the bad seeing it could be easily confused with the host with $R \sim 20$. Thus, we conclude that the burst had a $\beta_{\mathrm{OX}} \lesssim 0.5$ and therefore it can be considered a marginal member of the dark GRB population according to the Jakobsson et al. (2004) criterion. 


\section{Appendix B: Photometry}

Table B.1. Observation $\log$ for the GRB 050219A field.

\begin{tabular}{|c|c|c|c|c|c|c|c|}
\hline Filter & $\begin{array}{l}\text { Wavelength } \\
(\mu \mathrm{m})\end{array}$ & Telesc./Instr. & $\begin{array}{l}\text { Magnitudes } \\
\text { (Observed) }\end{array}$ & $\begin{array}{l}\text { AB Magnitudes } \\
\text { (Ext. cor.) }\end{array}$ & $\begin{array}{l}\text { Flux density } \\
(\mu \mathrm{Jy})\end{array}$ & $\begin{array}{l}\text { Upper } \\
(\mu \mathrm{Jy})\end{array}$ & $\begin{array}{c}\text { Lower } \\
(\mu \mathrm{Jy})\end{array}$ \\
\hline$F U V$ & 0.152 & GALEX & $>21.6$ & $>20.5$ & $<23.89$ & - & - \\
\hline$N U V$ & 0.193 & GALEX & $>21.7$ & $>20.6$ & $<20.99$ & - & - \\
\hline$u v w 2$ & 0.225 & Swift/UVOT & $>21.3$ & $>21.8$ & $<7.19$ & - & - \\
\hline uvm2 & 0.227 & Swift/UVOT & $>20.5$ & $>20.7$ & $<18.86$ & - & - \\
\hline$u v w 1$ & 0.260 & Swift/UVOT & $>21.3$ & $>21.7$ & $<7.42$ & - & - \\
\hline$u$ & 0.347 & Swift/UVOT & $>21.4$ & $>21.6$ & $<8.56$ & - & - \\
\hline$b$ & 0.430 & Swift/UVOT & $>21.7$ & $>20.8$ & $<16.90$ & - & - \\
\hline$v$ & 0.543 & Swift/UVOT & $20.82 \pm 0.12$ & 20.31 & 27.86 & 31.12 & 24.95 \\
\hline$g^{\prime}$ & 0.459 & $2.2 \mathrm{~m} / \mathrm{GROND}$ & $21.49 \pm 0.04$ & 20.85 & 16.96 & 17.64 & 16.38 \\
\hline$r^{\prime}$ & 0.622 & $2.2 \mathrm{~m} / \mathrm{GROND}$ & $20.17 \pm 0.04$ & 19.76 & 46.20 & 47.72 & 44.74 \\
\hline$i^{\prime}$ & 0.764 & $2.2 \mathrm{~m} / \mathrm{GROND}$ & $19.70 \pm 0.04$ & 19.38 & 65.55 & 67.89 & 63.30 \\
\hline$z^{\prime}$ & 0.899 & $2.2 \mathrm{~m} / \mathrm{GROND}$ & $19.41 \pm 0.04$ & 19.18 & 78.77 & 81.58 & 76.06 \\
\hline$J$ & 1.240 & $2.2 \mathrm{~m} / \mathrm{GROND}$ & $17.74 \pm 0.04$ & 18.52 & 145.06 & 149.82 & 140.46 \\
\hline$H$ & 1.647 & $2.2 \mathrm{~m} / \mathrm{GROND}$ & $17.01 \pm 0.10$ & 18.30 & 178.52 & 195.74 & 162.81 \\
\hline$K_{\mathrm{S}}$ & 2.170 & $2.2 \mathrm{~m} / \mathrm{GROND}$ & $16.66 \pm 0.12$ & 18.44 & 156.30 & 174.56 & 139.94 \\
\hline$R$ & 0.660 & VLT/FORS2 & $20.2 \pm 0.1$ & 20.0 & 37.19 & 33.91 & 40.78 \\
\hline $3.6 \mu \mathrm{m}$ & 3.543 & Spitzer/IRAC & $19.10 \pm 0.02$ & 19.10 & 85.00 & 87.00 & 83.00 \\
\hline $5.8 \mu \mathrm{m}$ & 5.711 & Spitzer/IRAC & $19.5 \pm 0.2$ & 19.5 & 58.00 & 66.00 & 50.00 \\
\hline $24 \mu \mathrm{m}$ & 23.68 & Spitzer/MIPS & $20.7 \pm 0.3$ & 20.7 & 19.00 & 24.00 & 14.00 \\
\hline 100 & 100 & Herschel/PACS & $>14.6$ & $>14.6$ & $<5400$ & - & - \\
\hline 160 & 160 & Herschel/PACS & $>14.4$ & $>14.4$ & $<15600$ & - & - \\
\hline 250 & 250 & Herschel/SPIRE & $>13.4$ & $>13.4$ & $<16500$ & - & - \\
\hline 350 & 350 & Herschel/SPIRE & $>13.1$ & $>13.1$ & $<21000$ & - & - \\
\hline 500 & 500 & Herschel/SPIRE & $>12.9$ & $>12.9$ & $<24600$ & - & - \\
\hline
\end{tabular}

Notes. Column 4 gives observed magnitudes and their errors in the native system of the filters. Column 5 has AB magnitudes corrected for Galactic extinction $(E(B-V)=0.16 \mathrm{mag})$. The last 3 columns are the corresponding flux densities and their upper and lower value in $\mu \mathrm{Jy}$ after correction for Galactic extinction. The upper limits are $3 \sigma$ above the background. To convert UVOT magnitudes into the AB system we used the following conversion: $u v w 2=+1.73$, uvm $2=+1.69$, $u v w 1=+1.51, u=+1.02, b=+0.13, v= \pm 0.01$. For the FORS2/R-band we used $R=+0.23$.

Table B.2. Secondary standard stars within 4 arcmin of the afterglow position (Fig. B.1).

\begin{tabular}{ccccccccc}
\hline \hline$\#$ & RA, Dec (J2000) & $g^{\prime}$ & $r^{\prime}$ & $i^{\prime}$ & $z^{\prime}$ & $J$ & $H$ \\
\hline 1 & $11: 05: 30.50,-40: 41: 53.7$ & $19.03(1)$ & $18.06(1)$ & $17.70(2)$ & $17.53(1)$ & $16.19(11)$ & $15.50(12)$ & $15.58(24)$ \\
2 & $11: 05: 37.16,-40: 41: 37.7$ & $16.27(1)$ & $15.23(1)$ & $14.89(2)$ & $14.67(1)$ & $13.61(3)$ & $13.09(3)$ & $12.95(3)$ \\
3 & $11: 05: 37.48,-40: 40: 08.1$ & $18.97(1)$ & $17.90(1)$ & $17.55(2)$ & $17.32(1)$ & $16.21(12)$ & $15.54(14)$ & $15.61(21)$ \\
4 & $11: 05: 39.79,-40: 42: 07.8$ & $19.75(2)$ & $18.38(1)$ & $17.87(2)$ & $17.58(1)$ & $16.36(12)$ & $15.88(18)$ & $15.39(19)$ \\
5 & $11: 05: 45.80,-40: 40: 25.5$ & $15.95(1)$ & $15.17(1)$ & $14.93(2)$ & $14.74(1)$ & $13.77(2)$ & $13.36(03)$ & $13.21(3)$ \\
\hline
\end{tabular}

Notes. Numbers in parentheses give the photometric $1 \sigma$ statistical uncertainty of the secondary standards in units of ten milli-mag. 


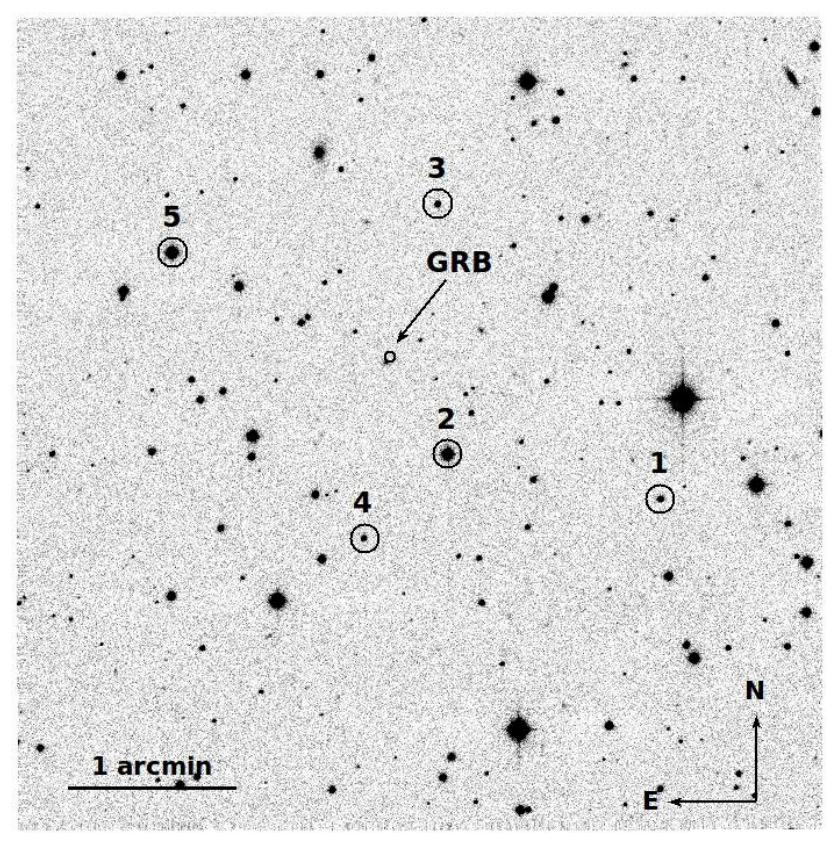

Fig. B.1. Finding chart of the field of GRB 050219A (GROND $r^{\prime}$-band). The X-ray afterglow position and the secondary photometric standards used (Table B.2) are indicated. 